\title{
ON COEFFICIENT INEQUALITIES FOR MEROMORPHIC UNIVALENT FUNCTIONS
}

\author{
LIQUAN LIU
}

(Communicated by Clifford J. Earle, Jr.)

\begin{abstract}
We obtain some coefficient inequalities for the class $\Sigma$ consisting of functions of the form $f(z)=z+b_{0}+b_{1} / z+\cdots$ that are meromorphic and univalent in the exterior of the unit circle $|z|=1$. These inequalities disprove two conjectures of Schober about linear functionals on $\Sigma$.
\end{abstract}

\section{INTRODUCTION}

Let $\Sigma$ denote the class of functions of the form

$$
f(z)=z+b_{0}+\frac{b_{1}}{z}+\frac{b_{2}}{z^{2}}+\frac{b_{3}}{z^{3}}+\cdots
$$

that are meromorphic and univalent in $\Delta=\{z:|z|>1\}$. Let $L$ be a complexvalued continuous linear functional on the space of all meromorphic functions in $\Sigma$, under the topology of uniform convergence on compact subsets. Suppose that $L$ is not constant on $\Sigma$ and that $L$ does not depend on the coefficient $b_{0}$ of $f$. We pose the extremal problem of maximizing $\operatorname{Re}\{L\}$ over $\Sigma$. The extremal functions are said to be support points of $\Sigma$ associated with the linear functional $L$.

It is well known (see [1, Theorem 10.1], [2], or [3, Chapter 10]) that every support point $f \in \Sigma$ maps $\Delta$ onto the complement of a continuum $\Gamma$ that consists of a finite number of analytic arcs satisfying the differential equation

$$
L\left(\frac{1}{f-w}\right) d w^{2} \geq 0 .
$$

Schober [4] posed the following two conjectures for general linear problems.

1. If $f$ is a support point of $\Sigma$ associated with a linear functional $L$, then $L\left(\frac{1}{f-w}\right)$ as a function of $w$ can have only simple zeros on the continuum $\Gamma$ omitted by $f$.

Received by the editors July 23, 1990; the contents of this paper have been presented to the Seventh Conference on Complex Analysis held in Qingdao, China on September 6, 1990.

1980 Mathematics Subject Classification (1985 Revision). Primary 30C50; Secondary 30C70, $30 \mathrm{C} 55$.

Key words and phrases. Univalent functions, coefficient inequalities, support points. 
2. For each $k \geq 2$ there is a function $f \in \Sigma$ so that

$$
\operatorname{Re}\left\{b_{k+1}+\sum_{j=1}^{k} \frac{j}{k+1}\left(\begin{array}{c}
2 k+2 \\
j+k+1
\end{array}\right) b_{j}\right\}>\frac{1}{k+1}\left(\begin{array}{c}
2 k+2 \\
k
\end{array}\right) .
$$

In particular, there exists a function in $\Sigma$ for which

$$
\operatorname{Re}\left\{b_{3}+4 b_{2}+5 b_{1}\right\}>5 \text {. }
$$

In this paper we prove the coefficient inequalities

$$
\operatorname{Re}\left\{b_{3}+4 b_{2}+5 b_{1}\right\} \leq 5, \quad \operatorname{Re}\left\{b_{3}+b_{2}+\frac{5}{4} b_{1}\right\} \leq \frac{5}{4},
$$

and

$$
\operatorname{Re}\left\{b_{3}+b_{1}\right\} \leq 1,
$$

with equalities holding only for

$$
f(z)=z+1 / z+\text { const } .
$$

The first inequality shows that conjecture 2 is not true for $k=2$.

Functions $f_{1}(z)=z+2+1 / z, f_{2}(z)=z+1+1 / z$, and $f_{3}(z)=z+1 / z$ are support points of $\Sigma$ associated with the linear functionals $L_{1}(f)=b_{3}+$ $4 b_{2}+5 b_{1}, L_{2}(f)=b_{3}+b_{2}+\frac{5}{4} b_{1}$, and $L_{3}(f)=b_{3}+b_{1}$ respectively. Now $L_{j}\left(\frac{1}{f_{j}-w}\right)=w^{2}, j=1,2,3$, have double zero $w=0$, which lies on $\Gamma_{1}=$ $\{w: 0 \leq w \leq 4\}, \Gamma_{2}=\{w:-1 \leq w \leq 3\}$, and $\Gamma_{3}=\{w:-2 \leq w \leq 2\}$ omitted, respectively, by $f_{1}, f_{2}$, and $f_{3}$. Each of these three examples shows that conjecture 1 is not true.

\section{SOME LEMMAS}

In order to derive our results, we first prove three lemmas, which may further be used to derive other coefficient inequalities involving $b_{1}, b_{2}$, and $b_{3}$.

Lemma 1. Let $f(z)=z+b_{0}+b_{1} / z+\cdots$ be a support point of $\Sigma$ associated with the linear functional $L$ for which

$$
L\left(\frac{1}{f-w}\right)=w^{2}+1-b_{1} .
$$

Then the omitted set $\Gamma$ of $f$ cannot contain both roots of $w^{2}=b_{1}-1$ unless $f$ is the function (3).

Proof. The differential equation for $\Gamma$ is now of the form

$$
\left(w^{2}+1-b_{1}\right) d w^{2} \geq 0 .
$$

If we put $b_{1}-1=\rho^{2} e^{i \alpha}, \alpha$ real, then $\frac{1}{2} \pi \leq|\alpha| \leq \pi$ since $\operatorname{Re}\left\{b_{1}\right\} \leq 1$. Now equation (5) can be written as

$$
e^{i 2 \alpha}\left(w e^{-i \frac{1}{2} \alpha}+\rho\right)\left(w e^{-i \frac{1}{2} \alpha}-\rho\right)\left(d\left(w e^{-i \frac{1}{2} \alpha}\right)\right)^{2} \geq 0 .
$$

Notice that the Schwarz-Christoffel transformation

$$
\zeta=\int_{0}^{w}(t+\rho)^{\frac{1}{2}}(t-\rho)^{\frac{1}{2}} d t
$$


maps the upper halfplane $\operatorname{Im}\{w\}>0$ onto the exterior of an infinite semistrip of the form

$$
\operatorname{Re}\{\zeta\}>0, \quad|\operatorname{Im}\{\zeta\}|<h,
$$

where

$$
h=\operatorname{Im}\left\{\int_{0}^{\rho}(t+\rho)^{\frac{1}{2}}(t-\rho)^{\frac{1}{2}} d t\right\} .
$$

Equation (6) merely states that $\Gamma$ is the pre-image by the transformation

$$
\zeta=\phi(w)=e^{i \alpha} \int_{0}^{w e^{-i \frac{1}{2} \alpha}}\left(t e^{-i \frac{1}{2} \alpha}+\rho\right)^{\frac{1}{2}}\left(t e^{-i \frac{1}{2} \alpha}-\rho\right)^{\frac{1}{2}} d\left(t e^{-i \frac{1}{2} \alpha}\right)
$$

of a line segment $\wedge$ in the $\zeta$-plane parallel to the real axis $\operatorname{Im}\{\zeta\}=0$. Since the points $\rho e^{-i \alpha / 2}$ and $-\rho e^{-i \alpha / 2}$ are on $\Gamma$, the corresponding points $i h e^{i \alpha}$ and $-i h e^{-i \alpha}$ are on $\wedge$ so that $|\alpha|=\frac{1}{2} \pi$ and $\operatorname{Re}\left\{b_{1}-1\right\}=0$. Hence $b_{1}=1$ and the conclusion of Lemma 1 follows.

Lemma 2. Under the hypotheses of Lemma 1, the omitted set $\Gamma$ by $f$ cannot omit both the points of $\left(b_{1}-1\right)^{1 / 2}$ and $-\left(b_{1}-1\right)^{1 / 2}$ unless $f$ is the function (3).

Proof. If we parametrize $\Gamma$ by $w=f\left(e^{i \theta}\right)$, then $d w / d \theta=i z f^{\prime}(z), z=e^{i \theta}$, and (5) becomes

$$
z^{2} f^{\prime}(z)^{2}\left[f(z)^{2}+1-b_{1}\right] \leq 0,
$$

where $|z|=1$. The left-hand side is an analytic function on $\Delta$ except for a single pole of order four at infinite. This function can be analytically continued into the interior of the unit circle by Schwarz reflection principle. In the neighborhood of infinity,

$$
\begin{aligned}
z^{2} f^{\prime}(z)^{2}\left[f(z)^{2}+1-b_{1}\right]= & z^{4}+2 b_{0} z^{3}+\left(b_{0}^{2}+1-b_{1}\right) z^{2}-2\left(b_{2}+b_{0} b_{1}\right) z \\
& -2\left(2 b_{3}+3 b_{0} b_{2}+b_{0}^{2} b_{1}+b_{1}\right)+\cdots
\end{aligned}
$$

It has a pole of order four at $z=0$. We may calculate it explicitly to obtain the differential equation

$$
z^{2} f^{\prime}(z)^{2}\left[f(z)^{2}+1-b_{1}\right]=R(z)
$$

where

$$
\begin{aligned}
R(z)= & z^{4}+2 b_{0} z^{3}+\left(b_{0}^{2}+1-b_{1}\right) z^{2}-2\left(b_{2}+b_{0} b_{1}\right) z \\
& -2\left(2 b_{3}+3 b_{0} b_{2}+b_{0}^{2} b_{1}+b_{1}\right) \\
& -\frac{2\left(\bar{b}_{2}+\bar{b}_{0} \bar{b}_{1}\right)}{z}+\frac{\bar{b}_{0}^{2}+1-\bar{b}_{1}}{z^{2}}+\frac{2 \bar{b}_{0}}{z^{3}}+\frac{1}{z^{4}} .
\end{aligned}
$$

If $b_{1} \neq 1$ then there are two distinct points $z_{1}$ and $z_{2}$ in $\Delta$ such that $f\left(z_{1}\right)=\left(b_{1}-1\right)^{1 / 2}$ and $f\left(z_{2}\right)=-\left(b_{1}-1\right)^{1 / 2}$. Then $z_{1}^{*}=z_{1}\left|z_{1}\right|^{-2}$ and $z_{2}^{*}=$ $z_{2}\left|z_{2}\right|^{-2}$ as well as $z_{1}$ and $z_{2}$ are simple zeros of $R(z)$ by (9) and the fact that $R(z)$ is real on $|z|=1$. Since $f^{\prime}(z)$ is free of zeros outside and inside the unit circle $|z|=1$, then either

(a) $f\left(z_{1}^{*}\right)=\left(b_{1}-1\right)^{1 / 2}, f\left(z_{2}^{*}\right)=-\left(b_{1}-1\right)^{1 / 2}$ or

(b) $f\left(z_{1}^{*}\right)=-\left(b_{1}-1\right)^{1 / 2}, f\left(z_{2}^{*}\right)=\left(b_{1}-1\right)^{1 / 2}$.

The composite function $\zeta=\phi(f(z))$, defined by (8), maps the circle $|z|=1$ 
onto $\wedge$ lying on a horizontal line $H$ in the $\zeta$-plane. By Schwarz reflection principle, $\zeta_{j}=\phi\left(f\left(z_{j}\right)\right)$ and $\zeta_{j}^{*}=\phi\left(f\left(z_{j}^{*}\right)\right), j=1,2$, are symmetric with respect to $H$. That is, ihe $e^{i \alpha}$ and $-i h e^{i \alpha}$ are on $H$ if (a) occurs and are symmetric with respect to $H$ if (b) occurs, where $h$ is given by (7).

In case (a) we must have $|\alpha|=\frac{1}{2} \pi$ and so $b_{1}=1$.

In case (b) we must have $\alpha=\pi$, by the previous choice of $\alpha$, and $H$ must be the real axis $\operatorname{Im}\{\zeta\}=0$. Thus $b_{1}=1$ again. The proof is complete.

Lemma 3. Under the hypotheses of Lemma 1 , if the omitted set $\Gamma$ by $f$ contains just one root of the equation $w^{2}+1-b_{1}=0$, then $b_{1}$ is real and there exists two numbers $r$ and $\eta$ with $r>1,|\eta|=1$ such that

$$
\begin{gathered}
\operatorname{Im}\left\{\left(1-3 r^{2}\right) \eta^{2}-2 b_{0} r \eta+2 \bar{b}_{0} \bar{\eta}+2 r \bar{\eta}^{2}\right\}=0, \\
1-b_{1}=\operatorname{Re}\left\{\left(1-3 r^{2}\right) \eta^{2}-2 b_{0} r \eta+2 \bar{b}_{0} \bar{\eta}+2 r \bar{\eta}^{2}\right\}, \\
\left(1-b_{1}\right) \log \frac{-(r-1)^{2} \eta^{2}}{1-b_{1}}+2 b_{0}^{2}+2 b_{0} r \eta+6 \bar{b}_{0} \bar{\eta}+4 r \bar{\eta}^{2}+4 b_{1}=0, \\
f\left(f^{2}+1-b_{1}\right)^{\frac{1}{2}}+\left(1-b_{1}\right) \log \left[f+\left(f^{2}+1-b_{1}\right)^{\frac{1}{2}}\right] \\
=\left(z+r \eta+2 b_{0}-\frac{r \bar{\eta}^{2}+2 \bar{b}_{0} \bar{\eta}}{z}-\frac{\bar{\eta}}{z^{2}}\right) Q-\frac{1-b_{1}}{2} \log \frac{-\eta^{2}}{1-b_{1}} \\
+\left(1-b_{1}\right) \log (Q+z-r \eta)-\log \left(1-\frac{Q+\eta}{z}\right),
\end{gathered}
$$

where $Q=\left(z^{2}-2 r \eta z+\eta^{2}\right)^{1 / 2}$.

Proof. The left-hand side of (9) now has only one zero in $\Delta$, say $\rho \eta,|\eta|=1$. Hence $R(z)$ has two simple zeros $\rho \eta$ and $\eta / \rho . R(z)$ has three double roots on the circle $|z|=1$ corresponding to the three tips of $\Gamma$. Therefore

$$
\begin{aligned}
R(z)= & \left(z+\alpha+\bar{\alpha} \bar{\eta} / z+\bar{\eta} / z^{2}\right)^{2}\left(z^{2}-2 r \eta z+\eta^{2}\right) \\
= & z^{4}+2(\alpha-r \eta) z^{3}+\left(\eta^{2}-4 \alpha r \eta+\alpha^{2}+2 \bar{\alpha} \bar{\eta}\right) z^{2} \\
& +2\left(\alpha \eta^{2}-2 r \bar{\alpha}-r \alpha^{2} \eta+\bar{\eta}+|\alpha|^{2} \bar{\eta}\right) z \\
& +\alpha^{2} \eta^{2}+\bar{\alpha}^{2} \bar{\eta}^{2}+2(\bar{\alpha} \eta+\alpha \bar{\eta})-4 r\left(1+|\alpha|^{2}\right)+\cdots+1 / z^{4},
\end{aligned}
$$

where $r=\frac{1}{2}(\rho+1 / \rho)$. Compare coefficients of (10) and (15),

$$
\begin{aligned}
b_{0} & =\alpha-r \eta, \\
b_{0}^{2}-b_{1}+1 & =\eta^{2}-4 \alpha r \eta+\alpha^{2}+2 \bar{\alpha} \bar{\eta} .
\end{aligned}
$$

Eliminating $\alpha$ between these two equalities leads to (11) and (12).

Equation (9) becomes

$$
z^{2} f^{\prime}(z)^{2}\left[f(z)^{2}+1-b_{1}\right]=\left(z+b_{0}+r \eta+\frac{\bar{b}_{0} \bar{\eta}+r \bar{\eta}^{2}}{z}+\frac{\bar{\eta}}{z^{2}}\right)^{2}\left(z^{2}-2 r \nu z+\eta^{2}\right),
$$

which can be rewritten as

$$
\left(f^{2}+1-b_{1}\right)^{\frac{1}{2}} d f=\left(1+\frac{b_{0}+r \eta}{z}+\frac{\bar{b}_{0} \bar{\eta}+r \bar{\eta}^{2}}{z^{2}}+\frac{\bar{\eta}}{z^{3}}\right)\left(z^{2}-2 r \eta z+\eta^{2}\right)^{\frac{1}{2}} d z .
$$


We introduce the notation

$$
Q=\left(z^{2}-2 r \eta z+\eta^{2}\right)^{\frac{1}{2}}
$$

and integrate the last differential equation to obtain

$$
\begin{aligned}
\frac{1}{2} f\left(f^{2}+1-b_{1}\right)^{\frac{1}{2}}+\frac{1-b_{1}}{2} \log \left[f+\left(f^{2}+1-b_{1}\right)^{\frac{1}{2}}\right]+K \\
=\frac{1}{2} Q\left(z+r \eta+2 b_{0}-\frac{r \bar{\eta}^{2}+2 \bar{b}_{0} \bar{\eta}}{z}-\frac{\bar{\eta}}{z^{2}}\right) \\
\quad+\frac{1-b_{1}}{2} \log (Q+z-r \eta)-\frac{1-\bar{b}_{1}}{2} \log \left(r-\frac{Q+\eta}{z}\right),
\end{aligned}
$$

where $K$ is a constant of integration.

In order to evaluate $K$, we substitute first $\rho \eta$ and then $\eta / \rho$ for $z$ into (16), using the fact $f(\rho \eta)=f(\eta / \rho)= \pm\left(b_{1}-1\right)^{1 / 2}=u$, say. We find successively that

$$
K+\frac{1-b_{1}}{2} \log u=\frac{1-b_{1}}{2} \log \frac{\eta}{2}\left(\rho-\frac{1}{\rho}\right)-\frac{1-\bar{b}_{1}}{2} \log \frac{1}{2}\left(\rho-\frac{1}{\rho}\right)
$$

and

$$
K+\frac{1-b_{1}}{2} \log u=\frac{1-b_{1}}{2} \log \frac{-\eta}{2}\left(\rho-\frac{1}{\rho}\right)-\frac{1-\bar{b}_{1}}{2} \log \frac{-1}{2}\left(\rho-\frac{1}{\rho}\right) .
$$

From these two equations we conclude that $b_{1}$ is real and so

$$
K=\frac{1-b_{1}}{2} \log \frac{\eta}{u}=\frac{1-b_{1}}{4} \log \frac{-\eta^{2}}{1-b_{1}} .
$$

From (16) and (17) we obtain (14).

On the other hand, $K$ can also be evaluated by expanding both sides of (16) about the point at infinity. This yields the relation

$$
\begin{aligned}
\frac{f^{2}}{2}- & \frac{1-b_{1}}{4}+\frac{1-b_{1}}{2} \log 2 f+K+0\left(\frac{1}{f}\right) \\
= & \frac{z^{2}}{2}+b_{0} z+\frac{\left(1-3 r^{2}\right) \eta^{2}}{4}-b_{0} r \eta-\bar{b}_{0} \bar{\eta}-\frac{r \bar{\eta}^{2}}{2} \\
& +\frac{1-b_{1}}{2} \log 2 z-\frac{1-b_{1}}{2} \log (r-1)+0\left(\frac{1}{z}\right) .
\end{aligned}
$$

Substituting for $f$ the expansion (1) and letting $z \rightarrow \infty$ we have

$$
K=\frac{1}{4}\left(1-3 r^{2}\right) \eta^{2}-b_{0} r \eta-\bar{b}_{0} \bar{\eta}-\frac{r \bar{\eta}^{2}}{2}-\frac{b_{0}^{2}}{2}-\frac{3 b_{1}+1}{4}-\frac{1-b_{1}}{2} \log (r-1)
$$

Now (13) follows from (17) and (18) and Lemma 3 is proved. 


\section{Coefficient INeQualities}

By means of the above three lemmas, we can prove the following theorems.

Theorem 1. Let the function (1) belong to $\Sigma$. Then

$$
\operatorname{Re}\left\{b_{3}+4 b_{2}+5 b_{1}\right\} \leq 5 \text {. }
$$

Equality holds only for the function (3).

Proof. Let $L(f)=b_{3}+4 b_{2}+5 b_{1}$, then $L$ is a linear functional over $\Sigma$. Since $L$ does not depend on the coefficient $b_{0}$, we may set $b_{0}=2$ so that (4) is satisfied. Let $f(z)=z+2+\cdots$ be a support point of $\Sigma$ associated with the functional $L$. The omitted set $\Gamma$ by $f$ satisfies the differential equation (5). It follows from Lemmas 1,2 , and 3 that if $f \neq z+2+1 / z$, then there exists $r>1$ and $\eta=e^{i \theta}, \theta$ real, such that (11)-(13) are satisfied. If we can show that such $r$ and $\eta$ do not exist, then our theorem is proved.

Substituting 2 for $b_{0}$ into (11), (12), and (13), we have, respectively,

$$
\begin{aligned}
& (1+r)(1-2 r) \sin 2 \theta-4(1+r) \sin \theta=0, \\
& x=(1-r)(1+3 r) \cos 2 \theta+4(1-r) \cos \theta,
\end{aligned}
$$

where $x=1-b_{1}$, and

$$
\frac{1-b_{1}}{2} \log \frac{-(r-1)^{2} \eta^{2}}{1-b_{1}}-2\left(1-b_{1}\right)+2 r \eta+6 \bar{\eta}+2 r \bar{\eta}^{2}+6=0 .
$$

Equation (19) has three solutions for $\theta$ in $(-\pi, \pi]: \theta=0, \pi$ and $\cos \theta=$ $2 /(1-3 r)$. If $\theta=0,(19)$ gives for $r>1$,

$$
x=5-2 r-3 r^{2}<0,
$$

and if $\theta=\pi,(19)$ becomes

$$
x=-3+6 r-3 r^{2}=-3(r-1)^{2}<0 .
$$

Both contradict $x=1-b_{1}>0$. If

$$
\cos \theta=2 /(1-3 r),
$$

equation (20) becomes

$$
x=\frac{3(1-r)^{2}\left(5+6 r+9 r^{2}\right)}{\left(1-3 r^{2}\right)} .
$$

Taking real part on the left-hand side of (21), we have

$$
\frac{x}{2} \log \frac{(r-1)^{2}}{x}-2 x+6+2(r+3) \cos \theta+2 r \cos 2 \theta=0 .
$$

Combining this with (22) and (23) and simplifying the results, we get

$$
\left(5+6 r+9 r^{2}\right)\left[2(1-r)^{2}+\log 3\left(5+6 r+9 r^{2}\right)\right]+6(r-1)=0 .
$$

Clearly, the expression in the left-hand side is positive for $r>1$ and hence this equation has no solution for $r>1$. The proof is completed.

Theorem 2. Let the function $f(z)$ belong to $\Sigma$. Then

$$
\operatorname{Re}\left\{b_{3}+b_{2}+\frac{5}{4} b_{1}\right\} \leq \frac{5}{4} \text {. }
$$


Equality occurs if and only if $f(z)$ is the function given by (3).

Proof. The support point

$$
f(z)=z+1+b_{1} / z+\cdots
$$

of $\Sigma$ associated with the functional $L(f)=b_{3}+b_{2}+\frac{5}{4} b_{1}$ maps $\Delta$ onto the $w$-plane omitting a set $\Gamma$, which satisfies the differential equation (5). Applying Lemmas 1 and 2 , we see that if $f \neq z+1+1 / z$, then $\Gamma$ contains just one root of the equation $w^{2}=b_{1}-1$. By Lemma 3, there exists $r>1$ and $\eta=e^{i \theta}, \theta$ real, such that (11)-(13) are satisfied. Since $b_{0}=1$, these equations become

$$
\begin{gathered}
2(1+r)[(1-2 r) \cos \theta-1] \sin \theta=0, \\
1-b_{1}=(1-r)(1+3 r) \cos 2 \theta-2 \cos \theta,
\end{gathered}
$$

and

$$
\frac{1-b_{1}}{2} \log \frac{(r-1)^{2} \eta^{2}}{1-b_{1}}-2\left(1-b_{1}\right)+3+r \eta+3 \bar{\eta}+2 r \bar{\eta}^{2}=0 .
$$

Equation (25) has three roots for $\theta$ in the interval $(-\pi, \pi]$.

If $\theta=0,(26)$ gives for $r>1$,

$$
1-b_{1}=(1-r)(3 r-1)<0,
$$

which is impossible.

If $\theta=\pi,(26)$ gives

$$
1-b_{1}=3(1-r)(1+r)<0
$$

for $r>1$, which is again impossible.

If

$$
\cos \theta=1 /(1-2 r),
$$

then (26) and (27) becomes, respectively,

$$
1-b_{1}=(r-1)\left(2-12 r-9 r^{2}+27 r^{3}\right) /(1-3 r)^{2}
$$

and

$$
\frac{1-b_{1}}{2} \log \frac{(r-1)^{2}}{1-b_{1}}-2\left(1-b_{1}\right)+\frac{3-8 r+9 r^{2}-18 r^{3}}{(1-3 r)^{2}}=0 .
$$

Eliminate $1-b_{1}$ between the last two equations to obtain

$$
\begin{aligned}
\frac{1}{2}(r-1)\left(2-12 r-9 r^{2}+27 r^{3}\right) \log \frac{(r-1)(1-3 r)^{2}}{2-12 r-9 r^{2}+27 r^{3}} \\
+7-36 r+15 r^{2}+54 r^{3}-54 r^{4}=0 .
\end{aligned}
$$

But for $r>1$ we have

$$
\begin{gathered}
(r-1)\left(2-12 r-9 r^{2}+27 r^{3}\right)>0, \\
(r-1)(1-3 r)^{2}<2-12 r-9 r^{2}+27 r^{3},
\end{gathered}
$$

and

$$
7-36 r+15 r^{2}+54 r^{3}-54 r^{4}<0
$$

Hence the expression on the left-hand side of (29) is negative over $r>1$, and so the equation (29) has no solution for $r>1$. This completes the proof of Theorem 2. 
Theorem 3. Let the function (1) be in $\Sigma$. Then

$$
\operatorname{Re}\left\{b_{3}+b_{1}\right\} \leq 1 \text {. }
$$

Equality holds only for the function (3).

Proof. Let $b_{0}=0$ and let

$$
f(z)=z+b_{1} / z+\cdots
$$

be a support point of $\Sigma$ associated with the linear functional $L(f)=b_{3}+b_{1}$. Then the omitted arcs $\Gamma$ by $f$ satisfy the differential equation (5). It follows from Lemmas 1 and 2 that if $f(z) \neq z+1 / z$, then $\Gamma$ contains one and only one root of the equation $w^{2}=b_{1}-1$. By Lemma 3 , there exists $r>1$ and $\eta=e^{i \theta}$, with $\theta$ real, such that $(11),(12)$, and (13) hold with $b_{0}=0$. That is,

$$
\begin{gathered}
(1+r)(1-3 r) \sin 2 \theta=0, \\
1-b_{1}=(1-r)(1+3 r) \cos 2 \theta,
\end{gathered}
$$

and

$$
\frac{1-b_{1}}{2} \log \frac{(r-1)^{2}}{1-b_{1}}-2\left(1-b_{1}\right)+2(1-r)=0 .
$$

The condition (30) yields $\sin 2 \theta=0$ so that (31) gives

$$
1-b_{1}=(r-1)(1+3 r)
$$

since $1-b_{1}>0$. Substituting this value for $1-b_{1}$ into (32), we obtain

$$
(1-r) \frac{1+3 r}{2} \log \frac{r-1}{1+3 r}-4-2 r+6 r^{2}=0 .
$$

But this equation has no solution on $1<r<+\infty$ as it is readily seen that the left-hand side is positive over $r>1$. Hence we must have $f(z)=z+1 / z$ and Theorem 3 is proved.

The extremal functions in Theorems 1, 2, and 3 are the same function (3). Now we provide an extremal problem whose extremal function differs from (3), although the omitted set satisfies the differential equation (5).

Theorem 4. Let the function (1) belong to $\Sigma$. Then

$$
\operatorname{Re}\left\{b_{3}+2 i b_{2}\right\} \leq \frac{1}{2} r^{3}-\frac{7}{2} r^{2}+4 r+1=1.138928 \ldots,
$$

where $r$ is the root of the equation

$$
\left(3 r^{2}-4 r+1\right) \log \frac{r-1}{3 r-1}-12 r^{2}+14 r+4=0 .
$$

Equality holds only for the function given by the implicit equation:

$$
\begin{aligned}
f\left(f^{2}+c\right)^{\frac{1}{2}}+c \log \left[f+\left(f^{2}+c\right)^{\frac{1}{2}}\right] \\
=\left[z-i(r-2)+\frac{r-2}{z}-\frac{i}{z^{2}}\right] Q+c \log c \\
\quad+\log (Q+z+i r)-\log \left(1-\frac{Q-i}{z}\right),
\end{aligned}
$$


where $c=3 r^{2}-4 r+1, Q=\left(z^{2}+2 i r z-1\right)^{1 / 2}$.

Proof. Let $b_{0}=i$ and let

$$
f(z)=z+i+b_{1} / z+\cdots
$$

be a support point of $\Sigma$ associated with the linear functional $L(f)=b_{3}+2 i b_{2}$. Then the omitted set $\Gamma$ by $f$ satisfies (5). Clearly $f \neq z+1 / z+i$. By Lemmas 1 and 2 the set $\Gamma$ contains one and only one root of $w^{2}=b_{1}-1$. By Lemma 3 we can find $r>1$ and $\eta=e^{i \theta}, \theta$ real, such that (11)-(14) are satisfied. That is, we have

$$
\begin{gathered}
2(r+1)[(1-3 r) \sin \theta-1] \cos \theta=0, \\
1-b_{1}=(r-1)[2 \sin \theta-(1-3 r) \cos 2 \theta], \\
\left(1-b_{1}\right) \log \frac{-(r-1)^{2} \eta^{2}}{1-b_{1}}+2-4\left(1-b_{1}\right)+2 i r \eta-6 i \bar{\eta}+4 r \bar{\eta}^{2}=0 .
\end{gathered}
$$

Equation (35) has three solutions: $\sin \theta=1 /(1-3 r), \theta=\pi / 2, \theta=-\pi / 2$.

If $\sin \theta=1 /(1-3 r),(36)$ becomes

$$
1-b_{1}=3(1-r)\left(9 r^{3}-3 r^{2}-r-1\right) /(3 r-1)^{2},
$$

which is negative for $r>1$. This contradicts $1-b_{1}>0$.

If $\theta=\pi / 2,(36)$ gives

$$
1-b_{1}=3 r^{2}+1>4 \text { for } r>1,
$$

which is impossible since $1-b_{1}<2$.

The only solution is hence $\theta=-\pi / 2$ or $\eta=-i$. Now (36) and (37) become, respectively,

$$
1-b_{1}=3 r^{2}-4 r+1
$$

and

$$
\left(1-b_{1}\right) \log \frac{(r-1)^{2}}{1-b_{1}}-4\left(1-b_{1}\right)-2 r+8=0 .
$$

Eliminating $1-b_{1}$ between these two equations we obtain (33). For $r>1$, (33) has only one root $r=1.29774029 \ldots$. Thus $r$ and $\eta=i$ are uniquely determined. With these values of $r$ and $\eta$, letting $b_{0}=i$ and using (38), we obtain from (14) the implicit equation for the extremal function $f(z)$.

To establish the inequality of the theorem, we have to represent $b_{3}+2 i b_{2}$ in term of $r$. Letting $b_{0}=i$ in (10) and $\alpha=i(1-r), \eta=-i$ in (15), we deduce

$$
\begin{aligned}
z^{4}+ & 2 i z^{3}-b_{1} z^{2}-2\left(b_{2}+i b_{1}\right) z-2\left(2 b_{3}+3 i b_{2}\right)+\cdots+1 / z^{4} \\
= & z^{4}+2 i z^{3}-r(3 r-4) z^{2}-2 i\left(1+r^{2}-r^{3}\right) z \\
& -2\left(2 r^{3}-5 r^{2}+4 r+1\right)+\cdots+1 / z^{4} .
\end{aligned}
$$

Comparing the coefficients of $z$ on both sides,

$$
b_{2}+i b_{1}=i\left(1+r^{2}-r^{3}\right) \text {. }
$$

Combining this with (38),

$$
i b_{2}=-r^{3}-2 r^{2}+4 r+1 .
$$


Identify the constant terms on both sides of (39) to obtain

$$
2 b_{3}+3 i b_{2}=2 r^{3}-5 r^{2}+4 r+1 .
$$

Thus we have

$$
b_{3}=\frac{5}{2} r^{3}+\frac{1}{2} r^{2}-4 r-1, \quad b_{3}+2 i b_{2}=\frac{1}{2} r^{3}-\frac{7}{2} r^{2}+4 r+1 .
$$

Numerically,

$$
b_{1}=-0.138572 \ldots, \quad i b_{2}=0.637140 \ldots, \quad b_{3}=0.115008 \ldots,
$$

and

$$
b_{3}+2 i b_{2}=1.389288 \ldots
$$

The proof is complete.

Remark. Since the centroid of $\Gamma$ omitted by $f$ given by (34) is $b_{0}=i, \Gamma$ must fork at the point $i\left(1-b_{1}\right)^{1 / 2}=(1.067 \ldots) i$ in three directions. One arc of $\Gamma$ is a segment lying on the imaginary axis with $i\left(1-b_{1}\right)^{1 / 2}$ as the lower end.

\section{REFERENCES}

1. P. L. Duren, Univalent functions, Springer-Verlag, New York, 1983.

2. L. Liu, Some inequalities derived from fundamental lemma concerning Schlicht functions, Acta Math. Sinica 7 (1957), 313-326. (Chinese)

3. G. Schober, Univalent functions-selected topics, Lecture Notes in Math., vol. 478, SpringerVerlag, Berlin, Heidelberg, and New York, 1975.

4. _ Some conjectures for the class $\Sigma$, in Topics in complex analysis, Contemp. Math., vol. 38, Amer. Math. Soc., Providence, R.I., 1985, pp. 13-21.

Department of Mathematics, Heilonguiang University, Harbin 150080, People's RePUBLIC OF CHINA 